\title{
Vertebral anti-fracture efficacy of strontium ranelate according to pre-treatment bone turnover
}

\author{
J. Collette • O. Bruyère • J. M. Kaufman • R. Lorenc • \\ D. Felsenberg • T. D. Spector • M. Diaz- Curiel • \\ S. Boonen • J.-Y. Reginster
}

Received: 7 November 2008 / Accepted: 31 March 2009/Published online: 13 May 2009

(C) The Author(s) 2009. This article is published with open access at Springerlink.com

\begin{abstract}
Summary Osteoporotic post-menopausal women patients in two randomised trials comparing the anti-fracture efficacy of strontium ranelate with placebo were separated into tertiles according to their baseline levels of biochemical markers of bone formation and resorption. The vertebral anti-fracture efficacy of strontium ranelate was shown to be independent of baseline bone turnover levels.

Introduction Bone turnover (BTO) levels vary among women at risk of osteoporotic fracture. Strontium ranelate is an anti-osteoporotic treatment increasing bone formation and reducing bone resorption. It was hypothesised that its anti-fracture efficacy would be independent of baseline BTO levels.
\end{abstract}

\section{J. Collette}

Department of Clinical Biology,

Bone and Cartilage Markers Laboratory, University of Liège,

Liège, Belgium

$\mathrm{O}$. Bruyère $\cdot$ J.-Y. Reginster

WHO Collaborating Centre for Public Health

Aspect of Osteoarticular Disorders, University of Liège,

Liège, Belgium

J. M. Kaufman

Department of Endocrinology and Unit for osteoporosis and Metabolic Bone Diseases, Ghent University Hospital, Ghent, Belgium

R. Lorenc

Department of Biochemistry, The Child's Health Center,

Warsaw, Poland

\section{Felsenberg}

Charité Campus Benjamin Franklin,

Centre of Muscle and Bone Research,

Free \& Humboldt University,

Berlin, Germany
Methods Post-menopausal women with osteoporosis from two pooled studies were stratified in tertiles according to baseline levels of two BTO markers: bone-specific alkaline phosphatase (b-ALP, $n=4995$ ) and serum C-telopeptide crosslinks (sCTX, $n=4891$ ). Vertebral fracture risk was assessed over 3 years with strontium ranelate $2 \mathrm{~g}$ /day or placebo.

Results In the placebo group, relative risk of vertebral fractures increased with BTO tertiles by $32 \%$ and $24 \%$ for patients in the highest tertile for b-ALP and CTX, respectively, compared to those in the lowest tertile. In the strontium ranelate group, incidences of vertebral fracture did not differ significantly across BTO tertiles. Significant reductions in vertebral fractures with strontium ranelate were seen in all tertiles of both markers, with relative risk reductions of $31 \%$ to

T. D. Spector

Department of Twin Research and Genetic Epidemiology,

Kings College London, St Thomas' Hospital Campus,

London, United Kingdom

M. Diaz- Curiel

Jimenez Diaz Foundation,

Madrid, Spain

S. Boonen

Division of Geriatric Medicine,

UZ Gasthuisberg,

Leuven, Belgium

J. Collette $(\square)$

CHU Liège,

Tour N²-B4000,

Sart Tilman, Belgium

e-mail: J.Collette@chu.ulg.ac.be 
$47 \%$ relative to placebo. Risk reduction did not differ among tertiles (b-ALP: $p=0.513$; sCTX: $p=0.290$ ).

Conclusion The vertebral anti-fracture efficacy of strontium ranelate was independent of baseline BTO levels. Strontium ranelate offers clinical benefits to women across a wide range of metabolic states.

Keywords Anti-fracture efficacy · Biochemical marker . Bone turnover · Osteoporosis · Strontium ranelate .

Vertebral fracture

\section{Introduction}

Osteoporosis is a complex disease, and many factors may contribute to the skeletal fragility that underlies osteoporotic fractures [1]. Two processes are thought to be particularly important in post-menopausal osteoporosis. First, during adult life, in both men and women, resorption of bone tends to exceed bone formation at each of the basic multicellular units that are responsible for bone remodelling. Secondly, relative oestrogen deficiency in women after the menopause increases the rate of bone remodelling, accelerating the net loss of bone [2,3]. During long-term treatment, anti-resorptive anti-osteoporotic agents act primarily by decreasing the rate of bone remodelling [4]. For example, during treatment with the bisphosphonate alendronate, some biochemical markers of bone resorption show a rapid decrease of $50 \%$ to $65 \%$ within 1 month of treatment. However, this is accompanied by a delayed decrease in markers of bone formation of approximately $50 \%$, which reaches a nadir between 6 and 12 months [5]. It might be predicted that baseline bone turnover rates could influence the effects of treatment with anti-resorptive and other antiosteoporotic agents. For example, anti-resorptive agents might be expected to be of greatest benefit to women with high levels of bone turnover, while bone formation agents might be most effective in women with low rates of bone formation.

Studies of the effects of anti-resorptive agents on bone mineral density (BMD) have generally shown larger treatment effects in women with high pre-treatment levels of bone turnover [6-10]. However, results for osteoporotic fracture risk have been less consistent [11, 12]. The effects of teriparatide, an agent that increases bone formation, on BMD were also greater in women with high bone turnover [13], but the reduction in the relative risk of osteoporotic fracture was independent of the pre-treatment bone turnover level [14].

Strontium ranelate is an oral anti-osteoporotic agent that reduces the risk of vertebral [15], non-vertebral and hip [16] fractures in post-menopausal osteoporotic women. Experiments in vitro and in animals [17, 18], as well as measurements of biochemical markers of bone turnover in osteoporotic women in a clinical trial [15], have shown that strontium ranelate simultaneously stimulates bone formation and reduces bone resorption, although individual effects are less pronounced than those induced by PTH or bisphosphonates. Two previous analyses have demonstrated that strontium ranelate reduces the risk to have a new vertebral fracture in patients with a wide range of osteoporosis severity: in osteopenic patients with and without previous fractures, in osteoporotic patients without prevalent vertebral fractures and in severe osteoporotic patients (at least two prevalent vertebral fractures) $[19,20]$. The purpose of the present study was to determine whether the efficacy of strontium ranelate in increasing lumbar BMD and reducing vertebral fracture risk in post-menopausal women is influenced by the pre-treatment level of biochemical markers of bone turnover, using data obtained over 3 years in two large placebo-controlled clinical trials, the Spinal Osteoporosis Therapeutic Intervention (SOTI) study [15] and the Treatment of Peripheral Osteoporosis (TROPOS) study [16]. Given the specific effects on bone turnover and its wide efficacy profile to date, we hypothesise that its efficacy would be independent of pre-treatment bone turnover levels.

\section{Methods}

The present analysis is based on pooled data on vertebral fractures and markers of pre-treatment bone turnover taken from two randomised, double-blind, placebo-controlled, international studies in post-menopausal women with osteoporosis, that demonstrated the anti-fracture efficacy of strontium ranelate $2 \mathrm{~g}$ /day. The SOTI study [15] was aimed at vertebral anti-fracture efficacy, and the TROPOS study [16] was aimed at peripheral (non-vertebral) fractures. However, vertebral fractures were evaluated in TROPOS as a pre-specified secondary endpoint in those women who had a spinal radiograph at baseline and at least one post-baseline.

Patients

Patients for both the SOTI and TROPOS studies were included initially in a common, open-label run-in study, the FIRST study [21]. Detailed inclusion criteria have been published previously $[15,16,21]$. In brief, for inclusion in FIRST, women had to be Caucasian, post-menopausal for at least 5 years, ambulatory and considered to be osteoporotic and at high risk of fracture. During FIRST, the calcium and vitamin D status of all women was assessed, and they were given daily supplements of up to $1,000 \mathrm{mg}$ of elemental calcium and up to 800 IU of vitamin D for a period of 2 weeks to 6 months. Supplementation doses and duration 
were adjusted for each patient according to their baseline calcium and 25-OH vitamin D status. After the run-in period, eligible women were proposed for enrolment in either the SOTI or TROPOS studies, and supplementation was continued at the same doses throughout the randomised treatment periods of both these studies. The SOTI study included women $\geq 50$ years of age with low lumbar BMD $\left(<0.840 \mathrm{~g} / \mathrm{cm}^{2}\right.$ measured with Hologic instruments, T-score $\leq-2.4$ ) and at least one prevalent vertebral fracture confirmed by spinal radiography. The TROPOS study included women with femoral neck BMD $<0.600 \mathrm{~g} / \mathrm{cm}^{2}$ and aged $\geq 74$ years or 70-74 years with one additional risk factor (history of osteoporotic fracture after menopause, residence in a retirement home, frequent falls or maternal history of osteoporotic fracture of the hip, spine or wrist).

\section{Study design and efficacy measurements}

Patients were randomised to receive strontium ranelate $2 \mathrm{~g}$ /day or placebo for 5 years (TROPOS) or 4 years followed by a 1-year treatment-switch period (SOTI). In both studies, main efficacy analyses were performed at 3 years, and the vertebral fracture data over 3 years were used for the present analysis. Baseline refers to the commencement of the SOTI and TROPOS studies, not the time of inclusion in FIRST.

Vertebral fractures were determined from radiographs taken at baseline and annually thereafter and were analysed in the same way in both studies. Radiographs were analysed by the semi-quantitative method of Genant et al. [22, 23], using a four-point grading scale: grade 0 - normal; grade $1-$ mild deformity (20-25\% decrease in at least one vertebral height); grade 2-moderate deformity (25-40\% decrease); and grade $3-$ severe deformity ( $>40 \%$ decrease). A new vertebral fracture was defined as a change from a nonfractured vertebra (grade 0) to a vertebra rated grade 1 or higher. All radiographs were analysed at a central facility (CEMO, France) blinded to treatment assignment but not to temporal sequence.

Lumbar L2-4 and femoral neck BMD were measured at baseline, and lumbar BMD was measured every 6 months post-baseline by dual-energy X-ray absorptiometry using Hologic devices. All scans were analysed centrally, and a programme of cross-calibration across centres was performed throughout both studies [24].

Blood samples were collected at baseline, 3 months, 6 months, and then every 6 months. Serum samples were stored at $-80^{\circ} \mathrm{C}$ and analysed centrally after a maximum 6 months period of storage (University of Liège, Belgium). Serum concentration of bone-specific alkaline phosphatase (bALP), a marker of bone formation, was measured by immunoradiometric assay (Tandem ${ }^{\circledR}$ Ostase ${ }^{\circledR}$ Beckman Coulter, San Diego, CA, USA). Serum concentration of C-telopeptide cross-links (sCTX), a marker of bone resorption, was measured using an enzyme- linked immunosorbent assay (Serum CrossLaps ${ }^{\circledR}$ ELISA-Nordic Bioscience Diagnostic, formerly Osteometer BioTech, Herlev, Denmark).

All the assays were performed in duplicate per batch of maximum 140 and 86 unknown serum samples for b-ALP and SCTX, respectively. If the $\mathrm{CV}$ on the duplicate measurement was higher than $15 \%$, the sample was re-assayed in a run control. In each assay run, two quality control samples (QCs) were assayed before and after the unknown samples. The assay run was validated if the $\mathrm{CV}$ on the duplicate measurement of a QC was lower or equal to $15 \%$, if the QCs results were in their respective 2SD ranges determined previously and if the difference between the results obtained before and after the unknown samples did not exceed $15 \%$.

Both clinical studies were conducted in accordance with the ethical principles stated in the Declaration of Helsinki, 1964, as revised in Hong Kong, 1989. The study protocol was approved by independent ethics committees in each country and/or centre. All patients gave written informed consent.

\section{Statistical analysis}

All analyses were performed in accordance with the intention-to-treat principle: The population included all patients having a baseline and post-baseline lumbar X-ray and having a baseline value for b-ALP or SCTX.

Groups were compared at baseline on the lumbar and femoral BMD and corresponding T-scores using an ANOVA analysis, adjusted or not on age.

Vertebral fracture risk was assessed as the number of patients with at least one new osteoporotic vertebral fracture, analysed by the Kaplan-Meier method. Patients were stratified into tertiles of baseline (pre-treatment) levels of b-ALP and sCTX. The boundaries of the tertiles and the normal ranges for b-ALP and sCTX are given in Table 1. Between-treatment differences in vertebral fracture risk over 3 years for each tertile were assessed using an unadjusted Cox model. Sensitivity analysis was performed using a Cox model adjusted for baseline lumbar BMD.

Table 1 Tertile boundaries and normal ranges for markers of bone turnover (b-ALP and sCTX)

\begin{tabular}{llll}
\hline & Tertile 1 & Tertile 2 & Tertile 3 \\
\hline $\mathrm{b}-\mathrm{ALP}(\mu \mathrm{g} / \mathrm{L})^{\mathrm{a}}$ & $\leq 10.0$ & $>10.0-\leq 13.3$ & $>13.3$ \\
$\operatorname{sCTX~}(\mathrm{ng} / \mathrm{mL})^{\mathrm{b}}$ & $\leq 0.423$ & $>0.423-\leq 0.626$ & $>0.626$ \\
\hline
\end{tabular}

${ }^{a}$ b-ALP, bone-specific alkaline phosphatase: normal range, 2.9-14.5 $\mu \mathrm{g} / \mathrm{L}$ (premenopausal women); 3.8-22.6 $\mu \mathrm{g} / \mathrm{L}$ (post-menopausal women)

${ }^{\mathrm{b}} \mathrm{SCTX}$, serum C-telopeptide cross-links: normal range, 0.112 $0.323 \mathrm{ng} / \mathrm{mL}$ (pre-menopausal women); $0.153-0.625 \mathrm{ng} / \mathrm{mL}$ (postmenopausal women) 
Table 2 Patients' characteristics at baseline by tertiles of b-ALP and SCTX
Expressed as mean \pm standard deviation

$b-A L P$ bone-specific alkaline phosphatase, $B M D$ bone mineral density, $s C T X$ serum

C-telopeptide cross-links

\begin{tabular}{llll}
\hline & Tertile 1 & Tertile 2 & Tertile 3 \\
\hline According to b-ALP level & $n=1,683$ & $n=1,642$ & $n=1,630$ \\
Age (years) & $74.5 \pm 6.2$ & $73.7 \pm 6.3$ & $73.8 \pm 6.0$ \\
Lumbar BMD (g/cm ${ }^{2}$ ) & $0.792 \pm 0.146$ & $0.781 \pm 0.148$ & $0.760 \pm 0.149$ \\
Lumbar BMD T-score & $-2.9 \pm 1.5$ & $-3.0 \pm 1.5$ & $-3.2 \pm 1.6$ \\
Mean number of prevalent vertebral fractures & $2.5 \pm 2.2$ & $2.5 \pm 2.2$ & $2.6 \pm 2.3$ \\
Femoral neck BMD $\left(\mathrm{g} / \mathrm{cm}^{2}\right.$ ) & $0.573 \pm 0.072$ & $0.569 \pm 0.073$ & $0.560 \pm 0.073$ \\
Femoral neck T-score & $-2.9 \pm 0.7$ & $-3.0 \pm 0.7$ & $-3.1 \pm 0.7$ \\
Mean number of previous peripheral fractures & $1.6 \pm 0.9$ & $1.6 \pm 0.9$ & $1.6 \pm 0.9$ \\
According to sCTX level & $n=1,631$ & $n=1,630$ & $n=1,630$ \\
Age (years) & $73.6 \pm 6.2$ & $73.9 \pm 6.3$ & $74.4 \pm 6.0$ \\
Lumbar BMD (g/cm ${ }^{2}$ ) & $0.798 \pm 0.149$ & $0.778 \pm 0.150$ & $0.755 \pm 0.145$ \\
Lumbar BMD T-score & $-2.8 \pm 1.5$ & $-3.0 \pm 1.6$ & $-3.3 \pm 1.5$ \\
Mean number of prevalent vertebral fractures & $2.6 \pm 2.3$ & $2.5 \pm 2.2$ & $2.5 \pm 2.2$ \\
Femoral neck BMD $\left(\mathrm{g} / \mathrm{cm}^{2}\right.$ ) & $0.579 \pm 0.075$ & $0.567 \pm 0.070$ & $0.556 \pm 0.072$ \\
Femoral neck T-score & $-2.9 \pm 0.7$ & $-3.0 \pm 0.6$ & $-3.1 \pm 0.6$ \\
Mean number of previous peripheral fractures & $1.6 \pm 0.9$ & $1.6 \pm 0.9$ & $1.6 \pm 1.0$ \\
\hline
\end{tabular}

Further between-treatment comparisons, using the same model, were performed for those patients who were in the lowest tertile for both b-ALP and sCTX (representing patients with the lowest bone turnover) and for patients in the highest tertile for both b-ALP and sCTX (representing those with the highest bone turnover).

\section{Results}

Patients

Of the 6,740 patients randomised in the two studies $(1,649$ in SOTI, 5,091 in TROPOS), 5,082 had a lumbar spinal radiograph at baseline and one or more post-baseline radiograph. Of these patients, 4,955 had a baseline measurement of b-ALP, and 4,891 patients had a baseline measure- ment of sCTX. These patients were then stratified into tertiles of b-ALP ( $n=1,683$ in tertile $1, n=1,642$ in tertile 2 and $1,630$ in tertile 3$)$ or $\operatorname{sCTX}(n=1,631$ in tertile $1, n=1,630$ in tertile 2 and $n=1,630$ in tertile 3 ). Baseline characteristics of patients, stratified into tertiles of baseline b-ALP and sCTX, are shown in Tables 2 and 3. The mean age of patients was approximately 74 years. Most variables were similar across tertiles. However, there were significant progressive reductions in lumbar and femoral neck BMD (ANOVA, $p<0.001$ for both sites), most obvious in the T-scores, with increasing tertiles of b-ALP and sCTX. There were no other relevant differences in baseline characteristics between tertiles, including in the levels of $25 \mathrm{OH}$ vitamin $\mathrm{D}$, creatinine or PTH. Regarding treatment group differences, baseline characteristics were similar in the strontium ranelate and placebo groups regardless of the tertile considered (as an example, lumbar BMD values are described in Table 3).

Table 3 Lumbar BMD values at baseline by tertiles of b-ALP and SCTX and treatment

\begin{tabular}{|c|c|c|c|c|c|c|}
\hline & \multicolumn{3}{|c|}{ Strontium ranelate } & \multicolumn{3}{|l|}{ Placebo } \\
\hline & Tertile 1 & Tertile 2 & Tertile 3 & Tertile 1 & Tertile 2 & Tertile 3 \\
\hline \multicolumn{7}{|l|}{ b-ALP } \\
\hline Lumbar BMD $\left(\mathrm{g} / \mathrm{cm}^{2}\right)$ & $0.793 \pm 0.140$ & $0.781 \pm 0.153$ & $0.759 \pm 0.152$ & $0.790 \pm 0.153$ & $0.781 \pm 0.143$ & $0.760 \pm 0.146$ \\
\hline T-score & $-2.8 \pm 1.5$ & $-2.9 \pm 1.6$ & $-3.2 \pm 1.6$ & $-2.9 \pm 1.6$ & $-3.0 \pm 1.5$ & $-3.2 \pm 1.5$ \\
\hline \multicolumn{7}{|l|}{ sCTX } \\
\hline Lumbar BMD $\left(\mathrm{g} / \mathrm{cm}^{2}\right)$ & $0.797 \pm 0.145$ & $0.780 \pm 0.153$ & $0.755 \pm 0.148$ & $0.800 \pm 0.153$ & $0.776 \pm 0.146$ & $0.755 \pm 0.142$ \\
\hline T-score & $-2.8 \pm 0.5$ & $-3.0 \pm 1.6$ & $-3.3 \pm 1.5$ & $-2.8 \pm 1.6$ & $-3.0 \pm 1.5$ & $-3.3 \pm 1.5$ \\
\hline
\end{tabular}

Expressed as mean \pm standard deviation

$b$ - $A L P$ bone-specific alkaline phosphatase, $B M D$ bone mineral density, $s C T X$ serum C-telopeptide cross-links 
Table 4 Incidence of vertebral fracture over 3 years of treatment with strontium ranelate (SR) compared with placebo, according to tertiles of pretreatment b-ALP and SCTX level

$C I$ confidence interval, $N N T$ number needed to treat

${ }^{\text {a }}$ Total number of patients having at least one new vertebral fracture during the 3-year period

\begin{tabular}{|c|c|c|c|c|c|c|}
\hline & \multicolumn{2}{|c|}{ Tertile 1} & \multicolumn{2}{|c|}{ Tertile 2} & \multicolumn{2}{|c|}{ Tertile 3} \\
\hline & $\mathrm{SR}$ & Placebo & SR & Placebo & $\mathrm{SR}$ & Placebo \\
\hline \multicolumn{7}{|l|}{ By b-ALP level } \\
\hline Events $^{\mathrm{a}}$ & 114 & 155 & 107 & 175 & 115 & 203 \\
\hline Incidence $(\%)$ & 14.9 & 21.1 & 14.3 & 23.7 & 16.4 & 26.5 \\
\hline Relative risk $[95 \% \mathrm{CI}]$ & \multicolumn{2}{|c|}{$0.69[0.54 ; 0.88]$} & \multicolumn{2}{|c|}{$0.58[0.46 ; 0.74]$} & \multicolumn{2}{|c|}{$0.58[0.46 ; 0.73]$} \\
\hline$p$ value & \multicolumn{2}{|c|}{0.003} & \multicolumn{2}{|c|}{$<0.001$} & \multicolumn{2}{|c|}{$<0.001$} \\
\hline Relative risk reduction $(\%)$ & \multicolumn{2}{|l|}{31} & \multicolumn{2}{|l|}{42} & \multicolumn{2}{|l|}{42} \\
\hline Absolute risk reduction $(\%)$ & \multicolumn{2}{|l|}{6.2} & \multicolumn{2}{|l|}{9.4} & \multicolumn{2}{|l|}{10.2} \\
\hline NNT & \multicolumn{2}{|l|}{17} & \multicolumn{2}{|l|}{11} & \multicolumn{2}{|l|}{10} \\
\hline \multicolumn{7}{|l|}{ By sCTX level } \\
\hline Events $^{\mathrm{a}}$ & 105 & 153 & 122 & 181 & 103 & 195 \\
\hline Incidence $(\%)$ & 13.8 & 21.2 & 16.9 & 24.1 & 14.7 & 26.3 \\
\hline Relative risk $[95 \% \mathrm{CI}]$ & \multicolumn{2}{|c|}{$0.63[0.49 ; 0.81]$} & \multicolumn{2}{|c|}{$0.68[0.54 ; 0.85]$} & \multicolumn{2}{|c|}{$0.53[0.42 ; 0.67]$} \\
\hline$p$ value & \multicolumn{2}{|c|}{$<0.001$} & \multicolumn{2}{|c|}{$<0.001$} & \multicolumn{2}{|c|}{$<0.001$} \\
\hline Relative risk reduction (\%) & \multicolumn{2}{|l|}{37} & \multicolumn{2}{|l|}{32} & \multicolumn{2}{|l|}{47} \\
\hline Absolute risk reduction (\%) & \multicolumn{2}{|l|}{7.4} & \multicolumn{2}{|l|}{7.2} & \multicolumn{2}{|l|}{11.6} \\
\hline NNT & \multicolumn{2}{|l|}{14} & \multicolumn{2}{|l|}{14} & \multicolumn{2}{|l|}{9} \\
\hline
\end{tabular}

Vertebral anti-fracture efficacy

The incidence of new vertebral fractures among patients treated with placebo was higher in patients in the highest tertile than in patients in the lowest tertile $(26.5 \%$ vs $21.1 \%$ in b-ALP tertiles 3 and $1, p=0.010$, and $26.3 \%$ vs $21.2 \%$ in sCTX tertiles 3 and $1, p=0.043$, respectively). Compared with the low turnover group (tertile 1), the relative risk to have a new vertebral fracture in patients with a high bone turnover level was increased over 3 years by $32 \%$ when considering b-ALP $(\mathrm{RR}=1.32,95 \% \mathrm{CI}$ $[1.06 ; 1.62])$ and $24 \%$ when considering $\mathrm{SCTX}(\mathrm{RR}=1.24$, $95 \%$ CI $[1.00 ; 1.54])$. This result was confirmed when comparing the incidence of new vertebral fracture in placebo patients in the subset with the lowest tertile for both b-ALP and SCTX with placebo patients in the highest tertile for both $b$-ALP and $\operatorname{sCTX}(\mathrm{RR}=1.47$, 95\% CI [1.08; 1.97], $p=0.012$ ).

Strontium ranelate was associated with a reduction in the relative risk of vertebral fracture, relative to placebo, of $40 \% \quad(\mathrm{RR}=0.60,95 \%$ CI [0.53-0.70], $p<0.001)$. When patients were stratified by tertiles of baseline levels of bone turnover markers, significant RR reductions with strontium ranelate were seen in each tertile of b-ALP $(31 \%, 42 \%$ and $42 \%$ for tertiles 1,2 and 3 , respectively). The same results were observed for tertiles of SCTX, with RR reductions of $37 \%, 32 \%$ and $47 \%$ for tertiles 1 to 3 , respectively (Table 4, Fig. 1). The magnitudes of the treatment effects were not significantly different between tertiles (interaction test $p=$ 0.513 for b-ALP tertiles, $p=0.290$ for sCTX tertiles). Results were similar after adjustment on lumbar BMD.
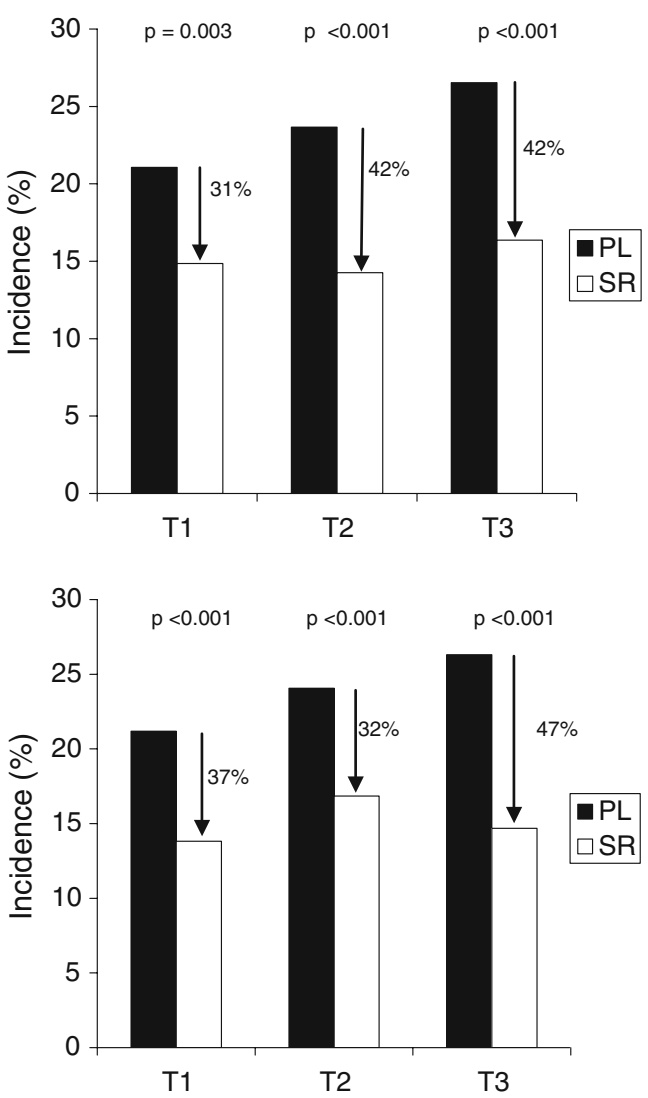

Fig. 1 Incidence of vertebral fractures over 3 years according to tertiles of b-ALP (upper panel) and sCTX (lower panel). SR strontium ranelate, $P L$ placebo 


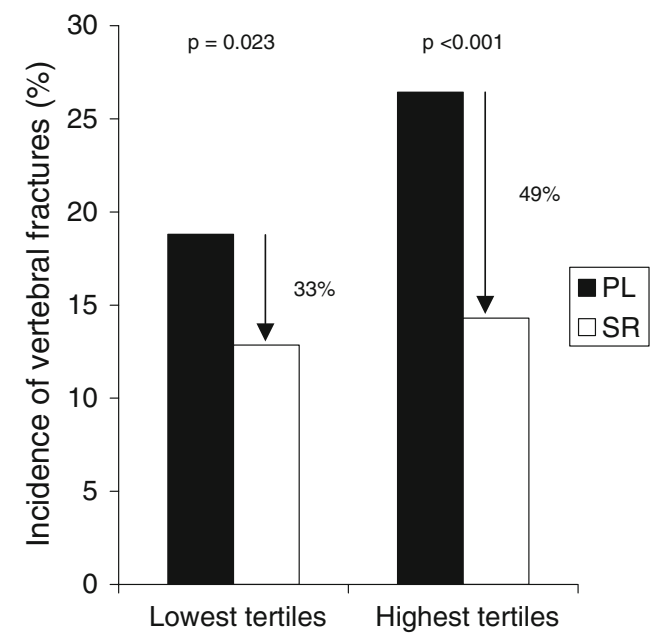

Fig. 2 Incidence of vertebral fractures over 3 years in patients in the lowest $(n=881)$ and highest $(n=867)$ tertiles for both b-ALP and sCTX. $S R$ strontium ranelate, $P L$ placebo

Among patients who were in the lowest tertile for both b-ALP and $\operatorname{sCTX}(n=881)$, strontium ranelate treatment produced a RR reduction of $33 \%$ compared to placebo ( $\mathrm{RR}=0.67,95 \%$ CI $[0.47 ; 0.95], p=0.023$; Fig. 2). Among patients in the highest tertile for both b-ALP and $\operatorname{sCTX}(n=867)$, the relative risk reduction with strontium ranelate was $49 \%(\mathrm{RR}=0.51,95 \%$ CI $[0.37 ; 0.70]$, $p<0.001)$. The fracture incidences in the strontium ranelate group were comparable, and the magnitude of the treatment effect was not significantly different between patients in the lowest and highest tertiles for both markers (interaction test $p=0.254$ ).

Given the increasing incidence of fractures with increasing bone turnover in patients treated with placebo, the absolute reduction in fracture risk with strontium ranelate was larger for higher tertiles of bone turnover markers. The number needed to treat (NNT) for 3 years to prevent one first new vertebral fracture ranged from 17 and 14 for the lowest tertiles of b-ALP and SCTX, respectively, to 10 and 9 for the highest tertiles (Table 4).

\section{Bone mineral density}

Lumbar BMD increased progressively during the 3-year analysis period in patients treated with strontium ranelate, but remained virtually unchanged in placebo-treated patients (Fig. 3). The increase in lumbar BMD with strontium ranelate, relative to baseline, at 3 years was $12.5 \%, 14.6 \%$ and $16.5 \%$ in b-ALP tertile 1, 2 and 3, respectively, and $12.6 \%$, $13.9 \%$ and $16.9 \%$ in sCTX tertile 1,2 , and 3, respectively $(p<0.001$ in all tertiles; Fig. 3). At each yearly time point, significant between-group differences in favour of strontium ranelate were observed in all tertiles $(p<0.001$ vs placebo at all time points for all tertiles of both b-ALP and SCTX).

\section{Discussion}

The main result from this analysis is that 3 years of treatment with strontium ranelate produced similar reductions in the risk of vertebral fracture, relative to placebo, in women with post-menopausal osteoporosis, irrespective of their baseline bone turnover level, consistent with our stated hypothesis. Substantial and significant reductions in fracture risk were seen across all tertiles of pre-treatment b-ALP (a marker of bone formation) and all tertiles of SCTX (a marker of bone resorption), and the size of the treatment effect did not differ significantly between tertiles of either biochemical marker. When women who were in the lowest tertile for both b-ALP and sCTX were compared with those in the highest tertile for both markers, significant relative risk reductions were seen in both groups, with a similar magnitude between the two groups. We further reported that in the placebo group, a high level of bone turnover at baseline is associated with a higher risk to have a new vertebral fracture in accordance to previous studies [25].

Bone turnover markers increase in women after the menopause. In one study, b-ALP, assayed using the same method as in the present study, was significantly higher in post-menopausal $(13.7 \mu \mathrm{g} / \mathrm{L})$ than pre-menopausal women $(10.8 \mu \mathrm{g} / \mathrm{L}, p<0.0001)$ [26]. Other studies have
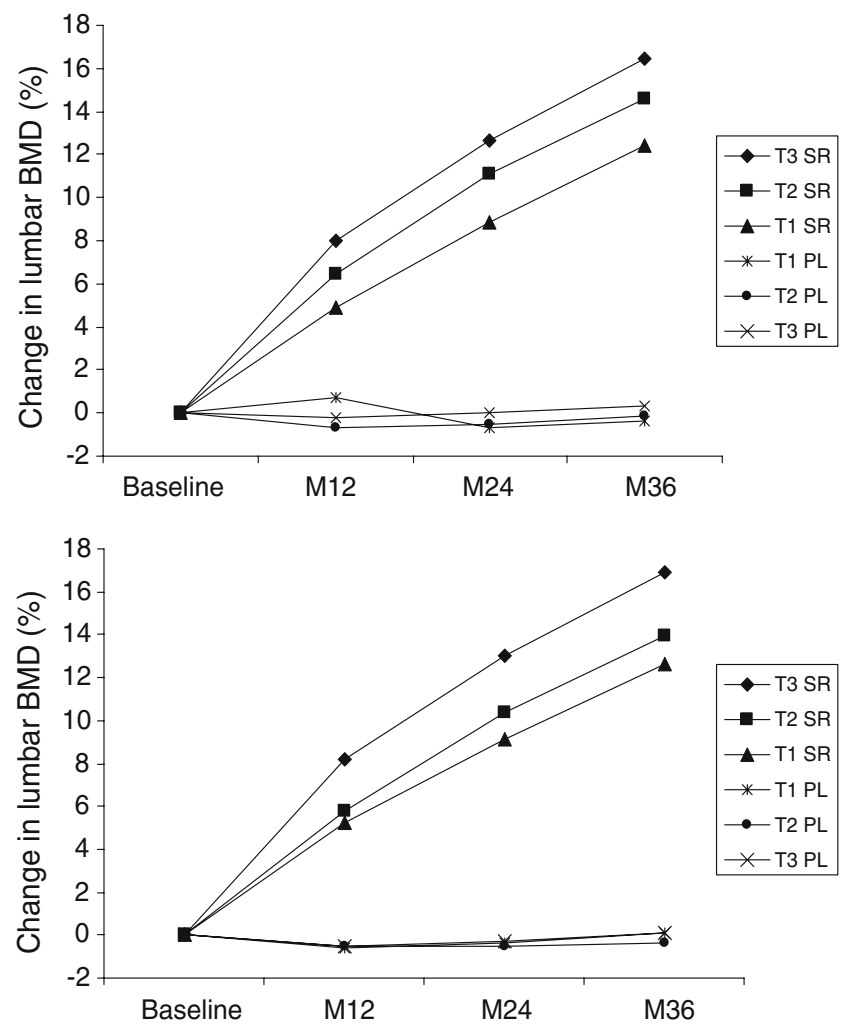

Fig. 3 Changes in lumbar bone mineral density (BMD) at 12, 24 and 36 months by tertiles of b-ALP (upper panel) and sCTX (lower panel) and treatment group. $S R$ strontium ranelate, $P L$ placebo 
found even lower values in healthy pre-menopausal women, of $8.2 \mu \mathrm{g} / \mathrm{L}$ [27] and $8.8 \mu \mathrm{g} / \mathrm{L}$ [28]. Reported mean values for post-menopausal women with osteoporosis range from approximately $12.5 \mu \mathrm{g} / \mathrm{L}$ [13] to $16.7 \mu \mathrm{g} / \mathrm{L}$ [27] and $18.1 \mu \mathrm{g} / \mathrm{L}$ [29]. The boundaries of the middle tertile for b-ALP in our sample were $>10.0$ and $\leq 13.3 \mu \mathrm{g} / \mathrm{L}$ and were slightly lower than the corresponding boundaries for osteoporotic subjects in the fracture intervention trial (FIT, 11.7 and $14.9 \mu \mathrm{g} / \mathrm{L}$ ) [12]. Regarding sCTX, levels in healthy premenopausal women have been measured at $1,748 \mathrm{pmol} / \mathrm{L}$ (corresponding to $0.225 \mathrm{ng} / \mathrm{mL}$ ) compared with $2,952 \mathrm{pmol} / \mathrm{L}$ (corresponding to $0.380 \mathrm{ng} / \mathrm{mL}$ ) in post-menopausal women [30]. Similarly, Garnero et al. [5] obtained levels of 0.299 and $0.556 \mathrm{ng} / \mathrm{mL}$ in pre- and post-menopausal women. The boundaries of the middle tertile for sCTX in our sample of post-menopausal osteoporotic women was $>0.423$ to $\leq 0.626 \mathrm{ng} / \mathrm{mL}$ (or 3,283 to $\leq 4,861 \mathrm{pmol} / \mathrm{L}$ ), slightly higher than in the FIT study $(2,337$ to $3,665 \mathrm{pmol} / \mathrm{L})$ [12]. Thus, the baseline levels of bone turnover markers in the present analysis are consistent with those in previous studies in postmenopausal women.

At baseline, higher tertiles of b-ALP and sCTX were associated with lower BMD, both at the lumbar spine and the femoral neck. Previous studies have reported that high bone turnover is correlated with low $\operatorname{BMD}[25,31]$ and predicts higher rates of future bone loss in post-menopausal women $[32,33]$. High bone turnover has also been associated with increased fracture risk, even after adjustment for BMD [31, $34,35]$. In our analysis, rates of prevalent vertebral and peripheral osteoporotic fractures at baseline did not differ between tertiles of bone turnover markers. However, the incidence of vertebral fractures during the study in the placebo group increased across ascending tertiles of both bone markers by $24 \%$ or more depending on the marker considered, with significant differences when comparing the lowest and highest tertiles (b-ALP or CTX independently or both b-ALP and CTX), suggesting that high bone turnover is a risk factor for fracture.

Strontium ranelate produced substantial increases in lumbar BMD independently of the baseline level of b-ALP or sCTX. Larger effects of treatment on BMD in women with higher baseline bone turnover level have been reported for many anti-osteoporotic drugs, including antiresorptive agents such as calcitonin [6], hormone replacement therapy [7] and bisphosphonates [8-10] and the bone formation agent, teriparatide [13]. Strontium ranelate treatment increased lumbar BMD in post-menopausal women across the range of baseline bone turnover and produced significant increases in BMD, relative to placebo, at each yearly time point in the present analysis.

The influence of baseline bone turnover level on the efficacy of anti-osteoporotic drugs on fracture risk has been less widely studied than BMD, and the results have been less consistent. In an analysis of a subgroup of 1,593 patients from three randomised trials of risedronate [11], vertebral antifracture efficacy was compared in women with baseline bone turnover levels, assessed by urinary excretion of deoxypyridinoline, above and below the normative median. At 3 years, the relative risk of vertebral fracture in patients with high bone turnover was 0.52 , similar to that in patients with low bone turnover (0.54). A recent analysis in 6,459 osteoporotic and non-osteoporotic women in the FIT study [12] concluded that the efficacy of alendronate in reducing non-vertebral fractures was greater in those with higher baseline bone turnover levels, although there was some inconsistency between different biochemical markers. The vertebral antifracture efficacy of alendronate was also influenced by baseline bone turnover in non-osteoporotic women, but no significant influence was found among osteoporotic women [12].

In the case of the bone formation agent, teriparatide, the relative risk reduction for osteoporotic fractures (vertebral and non-vertebral combined) was found to be similar for women in all tertiles of baseline bone turnover markers [14]. However, in that analysis, the risk of fracture increased markedly across tertiles of bone turnover markers, in both the placebo and teriparatide-treated groups. For example, the risks of fracture in the teriparatide group were $0.03,0.04$ and 0.08 in the low, middle and high tertiles of b-ALP, respectively. Thus, the absolute risk reduction with teriparatide was influenced by baseline bone turnover, and the number needed to treat to prevent one fracture decreased with higher tertiles of bone turnover markers. In the present study, the risk of fracture in the strontium ranelate group was similar across tertiles of baseline b-ALP and sCTX, whereas the fracture risk in women treated with placebo increased. The absolute reduction in fracture risk achieved with strontium ranelate treatment was therefore greater in women with higher pre-treatment bone turnover.

In a range of in vitro and in vivo experimental models, strontium ranelate has been shown to simultaneously reduce bone resorption and increase bone formation [18, 36, 37], without any change in bone mineralization [38-40]. Thus, strontium ranelate rebalances bone turnover in favour of bone formation. This effect of strontium ranelate on bone turnover may contribute to its anti-fracture efficacy in women with widely differing bone turnover status.

It is increasingly recognised that osteoporosis is a multifactorial disease. BMD is widely used both in diagnosis and fracture risk prediction. However, BMD accounts for only a modest proportion of fracture risk, and the majority of osteoporotic fractures occur in women who do not meet the World Health Organization definition of osteoporosis based on BMD (T-score -2.5 or less). Other clinical risk factors also contribute substantially to fracture risk [41, 42]. The recently introduced FRAX fracture risk assessment tool provides a framework for estimating fracture risk in individuals from clinical risk factors, including age, body mass index, previous fracture, parental history of fracture and 
current smoking, with or without the use of BMD [43]. A previous study demonstrated that the efficacy of a 3-year treatment with strontium ranelate on the risk of vertebral fractures is independent of baseline BMD and all of the above clinical risk factors [19]. The present analysis indicates that elevated levels of bone turnover markers is another risk factor for vertebral fracture and shows that the 3-year efficacy of strontium ranelate is also independent of the baseline bone turnover level. Three-year treatment with strontium ranelate therefore reduces vertebral fracture risk in post-menopausal women with a wide spectrum of risk factors for these fractures.

The main limitation of this study is that the results were based on post hoc analyses using pooled data from two studies with different entry criteria. However, both studies included women from a common run-in study (the FIRST study), and vertebral fracture, BMD and bone turnover data were collected using the same methodology. There were no significant differences in patients' characteristics at baseline between the strontium ranelate and placebo groups, and the only differences among patients in the tertiles of bone turnover markers are related to lumbar and femoral neck BMD. Pooling of data was therefore unlikely to have affected the conclusions of the study.

On the other hand, pooling of data allowed an adequate sample size and number of fractures to compare treatments after stratification of patients into tertiles and ensured that women with a wide range of disease severity and bone turnover were included in the analysis.

In conclusion, strontium ranelate showed significant vertebral anti-fracture efficacy in post-menopausal osteoporotic women in each tertile of markers of pre-treatment bone formation and resorption. The relative reductions in vertebral fracture risk achieved by strontium ranelate were independent of baseline bone turnover level. These results indicate that strontium ranelate offers clinical benefits to women across a wide range of metabolic states and disease severity.

Conflicts of interest Dr. Collette has no conflict of interest; Dr. Bruyère and Dr. Boonen received some consulting fees; Dr. Kaufman, Dr. Lorenc, Pr Felsenberg and Dr. Spector are investigators in SOTI and TROPOS studies; Pr Reginster received consulting fees, lecture fees and research grants from Servier.

Open Access This article is distributed under the terms of the Creative Commons Attribution Noncommercial License which permits any noncommercial use, distribution, and reproduction in any medium, provided the original author(s) and source are credited.

\section{References}

1. Bouxsein ML, Karasik D (2006) Bone geometry and skeletal fragility. Curr Osteoporos Rep 4:49-56

2. Seeman E (2003) The structural and biomechanical basis of the gain and loss of bone strength in women and men. Endocrinol Metab Clin North Am 32:25-38
3. Seeman E (2003) Invited review: pathogenesis of osteoporosis. J Appl Physiol 95:2142-2151

4. Stepan JJ, Alenfeld F, Boivin G et al (2003) Mechanisms of action of antiresorptive therapies of postmenopausal osteoporosis. Endocr Regul 37:227-240

5. Garnero P, Borel O, Delmas PD (2001) Evaluation of a fully automated serum assay for C-terminal cross-linking telopeptide of type I collagen in osteoporosis. Clin Chem 47:694-702

6. Civitelli R, Gonnelli S, Zachei F et al (1988) Bone turnover in postmenopausal osteoporosis. Effect of calcitonin treatment. J Clin Invest 82:1268-1274

7. Gonnelli S, Cepollaro C, Pondrelli C et al (1997) The usefulness of bone turnover in predicting the response to transdermal estrogen therapy in postmenopausal osteoporosis. J Bone Miner Res 12:624-631

8. Gonnelli S, Cepollaro C, Pondrelli C et al (1999) Boner turnover and the response to alendronate treatment in postmenopausal osteoporosis. Calcif Tissue Int 65:359-364

9. Iwamoto J, Takeda T, Sato Y et al (2004) Determinants of one-year response of lumbar bone mineral density to alendronate treatment in elderly Japanese women with osteoporosis. Yonsei Med J 45:676-682

10. Kim SW, Park DJ, Park KS et al (2005) Early changes in biochemical markers of bone turnover predict bone mineral density response to antiresorptive therapy in Korean postmenopausal women with osteoporosis. Endocr J 52:667-674

11. Seibel MJ, Naganathan V, Barton I et al (2004) Relationship between pretreatment bone resorption and vertebral fracture incidence in postmenopausal osteoporotic women treated with risedronate. J Bone Miner Res 19:323-329

12. Bauer DC, Garnero P, Hochberg MC et al (2006) Pretreatment levels of bone turnover and the antifracture efficacy of alendronate: the Fracture Intervention Trial. J Bone Miner Res 21:292-299

13. Chen P, Satterwhite JH, Licata AA et al (2005) Early changes in biochemical markers of bone formation predict BMD response to teriparatide in postmenopausal women with osteoporosis. J Bone Miner Res 20:962-970

14. Delmas PD, Licata AA, Reginster JY et al (2006) Fracture risk reduction during treatment with teriparatide is independent of pretreatment bone turnover. Bone 39:237-243

15. Meunier PJ, Roux C, Seeman E et al (2004) The effects of strontium ranelate on the risk of vertebral fracture in women with postmenopausal osteoporosis. New Engl J Med 350:459-468

16. Reginster JY, Seeman E, De Vernejoul MC et al (2005) Strontium ranelate reduces the risk of nonvertebral fractures in postmenopausal women with osteoporosis: Treatment of Peripheral Osteoporosis (TROPOS) study. J Clin Endocrinol Metab 90:2816-2822

17. Marie PJ, Ammann P, Boivin G et al (2001) Mechanisms of action and therapeutic potential of strontium in bone. Calcif Tissue Int 69:121-129

18. Marie PJ (2005) Strontium ranelate: a novel mode of action of optimizing bone formation and resorption. Osteoporos Int 16 (Suppl 1):S7-S10

19. Roux C, Reginster J-Y, Fechtenbaum J et al (2006) Vertebral fracture risk reduction with strontium ranelate in women with postmenopausal osteoporosis is independent of baseline risk factors. J Bone Miner Res 21:536-542

20. Seeman E, Devogelaer J-P, Lorenc R et al (2008) Strontium ranelate reduces the risk of vertebral fractures in patients with osteopenia. J Bone Miner Res 23:433-438

21. Meunier PJ, Reginster JY (2003) Design and methodology of the phase 3 trials for the clinical development of strontium ranelate in the treatment of women with postmenopausal osteoporosis. Osteoporos Int 14(Suppl 3):S66-S76

22. Genant HK, Wu CY, van Kuijk C et al (1993) Vertebral fracture assessment using a semiquantitative technique. J Bone Miner Res $8: 1137-1148$ 
23. Genant HK, Jergas M, Palermo L et al (1996) Comparison of semiquantitative visual and quantitative morphometric assessment of prevalent and incident vertebral fractures in osteoporosis. J Bone Miner Res 11:984-996

24. Slosman DO, Provvedini DM, Meunier PJ et al (1999) The use of different dual x-ray absorptiometry brands in a multicenter clinical trial. J Clin Densitom 2:37-44

25. Garnero P, Sornay-Rendu E, Chapuy MC et al (1996) Increased bone turnover in late postmenopausal women is a major determinant of osteoporosis. J Bone Miner Res 11:337-349

26. Broyles DL, Nielsen RG, Bussett EM et al (1998) Analytical and clinical performance characteristics of Tandem-MP Ostase, a new immunoassay for serum bone alkaline phosphatase. Clin Chem 44:2139-2147

27. Garnero P, Shih WJ, Gineyts E et al (1994) Comparison of new biochemical markers of bone turnover in late postmenopausal women in response to alendronate treatment. J Clin Endocrinol Metab 79:1693-1700

28. de Papp AE, Bone HG, Caulfield MP et al (2007) A crosssectional study of bone turnover markers in healthy premenopausal women. Bone 40:1222-1230

29. Bauer DC, Garnero P, Bilezikian JP et al (2006) Short-term changes in bone turnover markers and bone mineral density response to parthyroid hormone in postmenopausal women with osteoporosis. J Clin Endocrinol Metab 91:1370-1375

30. Rosenquist C, Fledelius C, Christgau S et al (1998) Serum CrossLaps One Step ELISA. First application of monoclonal antibodies for measurement in serum of bone-related degradation products from $\mathrm{C}$ terminal telopeptides of type I collagen. Clin Chem 44:2281-2289

31. Melton LJ 3rd, Khosla S, Atkinson EJ et al (1997) Relationship of bone turnover to bone density and fractures. J Bone Miner Res 12:1083-1091

32. Rogers A, Hannon RA, Eastell R (2000) Biochemical markers as predictors of rates of bone loss after menopause. J Bone Miner Res 15:1398-1404
33. Löfman O, Magnusson P, Toss G et al (2005) Common biochemical markers of bone turnover predict future bone loss: a 5-year follow-up study. Clin Chim Acta 356:67-75

34. Ravn P, Rix M, Andreassen H et al (1997) High bone turnover is associated with low bone mass and spinal fracture in postmenopausal women. Calcif Tissue Int 60:255-260

35. Garnero P, Sornay-Rendu E, Claustrat B et al (2000) Biochemical markers of bone turnover, endogenous hormones and the risk of fractures in postmenopausal women: the OFELY study. J Bone Miner Res 15:1526-1536

36. Buehler J, Chappuis P, Saffar JL et al (2001) Strontium ranelate inhibits bone resorption while maintaining bone formation in alveolar bone in monkeys (Macaca fascicularis). Bone 29:176-179

37. Bonnelye E, Chabadel A, Saltel F et al (2007) Dual effect of strontium ranelate: stimulation of osteoblast differentiation and inhibition of osteoclast formation and resorption in vitro. Bone 42:129-138

38. Ammann P, Shen V, Robin B et al (2004) Strontium ranelate improves bone resistance by increasing bone mass and improving architecture in intact female rats. J Bone Miner Res 19:12-20

39. Barbara A, Delannoy P, Denis BG et al (2004) Normal matrix mineralization induced by strontium ranelate in MC3T3-E1 osteogenic cells. Metabolism 53:532-537

40. Farlay D, Boivin G, Panczer G et al (2005) Long-term strontium ranelate administration in monkeys preserves characteristics of bone mineral crystals and degree of mineralization of bone. $\mathrm{J}$ Bone Miner Res 20:1569-1578

41. Leslie WD, Metge C, Ward L (2003) Contribution of clinical risk factors to bone density-based absolute fracture risk assessment in postmenopausal women. Osteoporos Int 14:334-338

42. Kanis JA, Oden A, Johnell O et al (2007) The use of clinical risk factors enhances the performance of BMD in the prediction of hip and osteoporotic fractures in men and women. Osteoporos Int 18:1033-1046

43. Kanis JA, Johnell O, Oden A et al (2008) FRAX and the assessment of fracture probability in men and women from the UK. Osteoporos Int 19:385-397 